\title{
ON THE KRYLOV MAXIMUM PRINCIPLE FOR DISCRETE PARABOLIC SCHEMES
}

\author{
HUNG-JU KUO AND NEIL S. TRUDINGER
}

Dedicated to Tamkang University on the occasion of its sixtieth anniversary

\begin{abstract}
In previous works, we have established discrete versions of the Krylov maximum principle for parabolic operators, on general meshes in Euclidean space. In this article, we prove a variant of these estimates in terms of a discrete analogue of the determinant of the coefficient matrix in the differential operator case. Our treatment adapts key ideas from our previous work on the corresponding discrete Aleksandrov maximum principle in the elliptic case.
\end{abstract}

\section{Introduction}

In our previous papers $[2,5]$, we provided a general discrete analogue of Krylov maximum principle [1] for linear second order parabolic partial differential operators in domains $\mathfrak{D}$ in Euclidean $(n+1)$-space $\mathbb{R}^{n+1}$. For operators $\widetilde{L}$ in the form

$$
\widetilde{L} u=D_{t} u-a^{i j}(x, t) D_{i j} u+b^{i}(x, t) D_{i} u+c(x, t) u
$$

acting on functions $u \in C^{2,1}(\mathfrak{D})$ with coefficient matrix $A=\left[a^{i j}\right]$ measurable and positive in $\mathfrak{D}$, the Krylov maximum principle provides an estimate,

$$
\sup _{\mathfrak{D}} u \leq C \operatorname{diam}(\mathfrak{D})^{\frac{n}{n+1}}\left\|\frac{\widetilde{L} u}{(\operatorname{det} A)^{1 /(n+1)}}\right\|_{L^{n+1}(\mathfrak{D})}
$$

where $C$ is a constant depending on $n$ and $b$ and $c$. In this article we extend our previous results to embrace the dependence of our estimates on the discrete analogue of the coefficient determinant, $\operatorname{det} A$, thereby establishing the parabolic analogue of our discrete Aleksandrov maximum principle in [6].

First we note that a general nonlinear parabolic difference equation may be written in the form,

$$
F[u](x, t):=F(x, t, u, T u)=0
$$

Corresponding author: Hung-Ju Kuo.

2000 Mathematics Subject Classification. Primary 65M06, 35K20, 39A70; Secondary 35A15, $65 \mathrm{M} 12,39 \mathrm{~A} 10$. 
where $u: E \rightarrow \mathbb{R}$ a mesh function defined on a space-time mesh $E$, which is a discrete subset of $(n+1)$-dimension Euclidean space, $\mathbb{R}^{n} \times \mathbb{R}$, with points denoted $(x, t), x \in$ $\mathbb{R}^{n}, t \in \mathbb{R}$. We shall refer to $x$ and $t$ as the spatial and time coordinates respectively. $F$ is a given real-valued function on $E \times \mathbb{R}^{E}$ and

$$
T u(x, t)=\{u(y, s) \mid(y, s) \neq(x, t)\} .
$$

We always assume that $F[u]$ is independent of the values $u(y, s)$ for $|y-x|$ sufficiently large and $s>t$. The operator $F$ is called monotone if

$$
F(x, t, u, q+\eta) \geq F(x, t, u, q)
$$

for all $(x, t) \in E, u \in \mathbb{R}, q \in \mathbb{R}^{E \backslash\{(x, t)\}}, \eta \geq 0$. If $F$ is differentiable with respect to $q_{z}$ then $F$ is monotone if $\frac{\partial F}{\partial q_{z}}(x, t, u, q) \geq 0$ for all $z=(y, s) \in E \backslash\{(x, t)\},(x, t) \in E$ and positive if in addition, $\sum_{z \in E} \frac{\partial F}{\partial q_{z}}(x, t, u, q) \leq 0$, for each $(x, t) \in E$. We also call $F$ spatially balanced if

$$
F(x, t, u, \widetilde{q})=F(x, t, u, q)
$$

whenever

$$
\widetilde{q}_{z}=q_{z}+p \cdot(y-x), \quad z=(y, s) \in E \quad \text { and for some } p \in \mathbb{R}^{n} .
$$

Let

$$
a^{\prime}(x, t ; y)=\sum_{\substack{z=(y, s) \\ \neq(x, t) \\ s \leq t}} \frac{\partial F}{\partial q_{z}}(x, t, u, q)
$$

and

$$
a^{i j}(x, t)=\frac{1}{2} \sum_{y} a^{\prime}(x, t ; y)(y-x)_{i}(y-x)_{j} .
$$

We call $F$ is spatially non-degenerate (or spatially elliptic) if $A=\left[a^{i j}\right]$ is positive that is

$$
\frac{1}{2} \sum_{\substack{z=(y, s) \\ \in \in}} \frac{\partial F}{\partial q_{z}}(x, t, u, q)[(y-x) \cdot \xi]^{2} \geq \lambda|\xi|^{2}
$$

for all $\xi \in \mathbb{R}^{n}$ and some positive constant $\lambda$. By virtue of the "linearization" formula

$$
\begin{aligned}
F[u](x, t)-F[v](x, t) & =L(u-v)(x, t) \\
& =\sum_{(y, s) \in E} a(x, t ; y, s)(u-v)(y, s)
\end{aligned}
$$

where

$$
a(x, t ; y, s)=\int_{0}^{1} \frac{\partial F}{\partial q_{z}}\left(x, t, w_{t}, T w_{t}\right) \mathrm{d} t, \quad z=(y, s)
$$


and

$$
w_{t}=w_{t}(x, t)=t u(x, t)+(1-t) v(x, t), \quad 0 \leq t \leq 1,
$$

where $u$ and $v$ are arbitrary mesh functions. Taking $v=0$ in (1.10), we may write the operator $F$ in the "linear" form

$$
F[u]=L u(x, t):=\sum_{(y, s) \in E} a(x, t ; y, s) u(y, s)+f(x, t)
$$

where

$$
f(x, t)=F(x, t, 0,0)
$$

with coefficients $a(x, t ; y, s)$ having finite support in $z$ for each $x \in E$. Accordingly, from (1.5), $L$ is of monotone type if

$$
a(x, t ; y, s) \geq 0 \quad \text { for all }(x, t, y, s) \in E \times E,(y, s) \neq(x, t)
$$

and of positive type if, in addition,

$$
\sum_{(y, s) \in E} a(x, t ; y, s) \leq 0 .
$$

The operator $L$ is then called evolving if

$$
a(x, t ; y, s)=0 \text { for } s>t,
$$

that is, its action is independent of values at future times. We also call $L$ spatially balanced if

$$
\begin{aligned}
b(x, t) & :=\sum_{(y, s)} a(x, t ; y, s)(x-y) \\
& =0
\end{aligned}
$$

and separately spatially balanced if

$$
\begin{aligned}
b^{\prime}(x, t, s) & :=\sum_{y} a(x, t ; y, s)(x-y) \\
& =0, \quad \text { for each } s \leq t .
\end{aligned}
$$

For each point $(x, t) \in E$, we define a spatial mesh $E^{\prime}=E^{\prime}(x, t)$ in $\mathbb{R}^{n}$, by

$$
E^{\prime}=\left\{y \in \mathbb{R}^{n} \mid a(x, t ; y, s)>0, \quad \text { for some } s \leq t\right\}
$$

and for $y \in E^{\prime}$, we define new coefficients $a^{\prime}$ by

$$
a^{\prime}(x, t, y)=\sum_{s \leq t} a(x, t ; y, s) .
$$


Moreover, let

$$
a^{i j}(x, t)=\frac{1}{2} \sum_{y} a^{\prime}(x, t ; y)(y-x)_{i}(y-x)_{j} \quad \text { and } \quad A=\left[a^{i j}\right] .
$$

A further discrete set $Z=Z(x, t)$ is then defined in $\mathbb{R}^{n}$ by

$$
Z(x, t)=\left\{x+a^{\prime}(x, t, y)(y-x) \mid y \in E^{\prime}(x, t)\right\} .
$$

Note that if $L$ is spatially balanced, then the set $Z$ is centered at $x$. We call the operator $L$ spatially non-degenerate ( or spatially elliptic ) if

$$
\frac{1}{2} \sum_{y} a^{\prime}(x, t ; y)[(y-x) \cdot \xi]^{2} \geq \lambda|\xi|^{2}
$$

for some positive constant $\lambda$. Further, we call $L$ time-wise non-degenerate if

$$
\gamma=\gamma(x, t):=\sum_{s<t} a(x, t ; x, s)>0
$$

To extend this condition to general space-time meshes, we call the operator $L$ of weak positive type if it is monotone and,

$$
\mu=\mu(x, t):=-\sum_{(y, t) \in E} a(x, t ; y, t)>0 .
$$

Note that $L$ is of positive type for sufficiently large $\mu$.

To illustrate the above conditions we recall the special case of a uniform mesh and single time step operator treated in [2]. Here the mesh $E$ is given by

$$
\begin{aligned}
E & =\mathbb{Z}_{h}^{n} \times \mathbb{Z}_{\tau} \\
& =\left\{(x, t) \in \mathbb{R}^{n+1} \mid x=\left(m_{1} \cdots, m_{n}\right) h, t=m \tau, m, m_{1} \cdots, m_{n} \in \mathbb{Z}\right\}
\end{aligned}
$$

where the spatial mesh length $h$ and time step $\tau$ are fixed positive constants. A spatial operator $L^{\prime}$ is defined on the cubic spatial mesh $\mathbb{Z}_{h}^{n} \subset \mathbb{R}^{n}$ by

$$
L^{\prime} u(x, t)=\sum_{z \neq 0} a(x, t ; z) \delta_{z}^{2} u(x, t)+b(x, t ; z) \delta_{z} u(x, t)+c(x, t) u(x, t)
$$

where the coefficients $a, b$ are real-valued functions on $E \times \mathbb{Z}_{h}^{n}$, vanishing for $|z|>K h$ for some $K \in \mathbb{N}, c$ is a real-valued function on $E$ and the difference operators $\delta_{z}, \delta_{z}^{2}$ are given by

$$
\begin{aligned}
& \delta_{z} u(x, t)=\frac{1}{2|z|}\{u(x+z, t)-u(x-z, t)\} \\
& \delta_{z}^{2} u(x, t)=\frac{1}{|z|^{2}}\{u(x+z, t)-2 u(x, t)+u(x-z, t)\} .
\end{aligned}
$$


The space-time difference operator $L$, corresponding to a standard explicit-implicit scheme, is then defined by

$$
L u(x, t)=(1-\alpha) L^{\prime} u(x, t)+\alpha L^{\prime} u(x, t-\tau)-\frac{1}{\tau}\{u(x, t)-u(x, t-\tau)\}
$$

where $\alpha$ is a fixed number satisfying $0 \leq \alpha \leq 1$. The scheme is implicit when $\alpha=0$ and explicit when $\alpha=1$. The operator $L$ is monotone if

$$
\begin{aligned}
& a\left(x, t^{\prime} ; z\right)-\frac{1}{2}|z|\left|b\left(x, t^{\prime} ; z\right)\right| \geq 0, \forall z \neq 0, \text { and } t^{\prime}=t, t-\tau \\
& \alpha \tau\left(2 \sum_{z \neq 0} \frac{a(x, t ; z)}{|z|^{2}}-c(x, t)\right) \leq 1 .
\end{aligned}
$$

The spatial non-degeneracy condition in [2] is that for each $(x, t) \in E$, there exists an orthogonal set of vectors $z^{1}, \cdots, z^{n} \in \mathbb{Z}_{h}^{n}$ such that

$$
\begin{aligned}
\lambda_{i}(x, t):= & (1-\alpha)\left\{a\left(x, t ; z^{i}\right)-\frac{\left|z^{i}\right|}{2}\left|b\left(x, t ; z^{i}\right)\right|\right\} \\
& +\alpha\left\{a\left(x, t-\tau ; z^{i}\right)-\frac{\left|z^{i}\right|}{2}\left|b\left(x, t-\tau ; z^{i}\right)\right|\right\}>0
\end{aligned}
$$

$i=1, \cdots, n$. It follows then that our present spatial non-degeneracy condition is that the coefficient matrix is positive

$$
A=\operatorname{diag}\left(\lambda_{1}, \cdots, \lambda_{n}\right)>0
$$

while the time-wise non-degeneracy condition (1.22) is satisfied for

$$
\gamma(x, t)=\frac{1}{\tau}-\alpha\left(2 \sum_{z \neq 0} \frac{a(x, t-\tau ; z)}{|z|^{2}}-c(x, t-\tau)\right)>0 .
$$

Finally the operator (1.27) is weakly positive if it is monotone and, instead of (1.12),

$$
\mu(x, t)=\frac{1}{\tau}-(1-\alpha) c(x, t)>0,
$$

in accordance with (1.23) above and [2].

\section{Preliminaries}

The proof of the maximum principle, Theorem 3.1 in next section, is based on certain inequalities of Krylov [1], together with the discrete adaptation in [6] of the geometric argument of Aleksandrov. As in our previous work [2], the sum on the right hand side of (3.4) can be taken over the increasing-upper contact set of $u$. As before, for a spatial 
mesh function $u$, defined on a subset $\mathfrak{D}^{\prime}$ of a discrete mesh $E^{\prime} \subset \mathbb{R}^{n}$, we define the upper contact set $\Gamma^{+}=\Gamma^{+}(u)$ by

$$
\begin{aligned}
\Gamma^{+}=\left\{x \in \mathfrak{D}^{\prime} \mid u(y) \leq u(x)+p \cdot(y-x)\right. & \text { for all } y \in \mathfrak{D}^{\prime}, \\
& \text { for some } \left.p \in \mathbb{R}^{n}\right\} .
\end{aligned}
$$

The spatial upper contact set of a space-time mesh function $u$ on a set $\mathfrak{D} \subset E$, is then defined by

$$
\Gamma^{+}=\Gamma^{+}(u)=\left\{(x, t) \in \mathfrak{D} \mid x \in \Gamma_{t}^{+}\right\},
$$

where $\Gamma_{t}^{+}$denotes the upper contact set on

$$
\mathfrak{D}_{t}=\left\{x \in \mathbb{R}^{n} \mid(x, t) \in \mathfrak{D}\right\}
$$

of the spatial mesh function $u^{t}$ given by $u^{t}(x)=u(x, t)$. The increasing set of a spacetime mesh function $u$ is defined by

$$
\begin{aligned}
I^{+} & =I^{+}(u) \\
& =\{(x, t) \in \mathfrak{D} \mid u(x, t)>u(x, s) \quad \text { for all }(x, s) \in \mathfrak{D}, s<t\},
\end{aligned}
$$

and the increasing upper contact set is then given by

$$
\mathcal{S}^{+}=\mathcal{S}^{+}(u)=\Gamma^{+} \cap I^{+} .
$$

Let $\mathfrak{D}$ be a bounded subset of the space-time mesh $E$, with $d, T$ denoting respectively the spatial and time diameters of $\mathfrak{D}$, that is

$$
\begin{aligned}
d & =\max \{|x-y| \mid(x, t),(y, s) \in \mathfrak{D}, \quad \text { for some } s, t \in \mathbb{R}\} \\
T & =\max \left\{t-s \mid(x, t),(y, s) \in \mathfrak{D}, \quad \text { for some } x, y \in \mathbb{R}^{n}\right\}
\end{aligned}
$$

Without loss of generality, we can assume that $\mathfrak{D}$ lies in the space-time cylinder $Q$ given by

$$
Q=B_{d / 2} \times[0, T] .
$$

For a fixed point $(x, t) \in \mathfrak{D}$, let us also define

$$
\tau=\tau(x, t)=\min \{t-s \mid(y, s) \in \mathfrak{D}, s<t, a(x, t ; y, s)>0\} .
$$

If $\mathfrak{D}$ is a subset of $E$, then the interior of $\mathfrak{D}$, with respect to $L$, is defined by

$$
\mathfrak{D}^{\circ}=\{(x, t) \in \mathfrak{D} \mid \quad a(x, t ; y, s)=0, \quad \forall \quad(y, s) \notin \mathfrak{D}\}
$$

and the boundary of $\mathfrak{D}$, with respect to $L$, is defined by

$$
\mathfrak{D}^{b}=\mathfrak{D}-\mathfrak{D}^{\circ} .
$$

The basic inequalities we need are encompassed in the following lemmas (also see [2] ), which correspond to special cases of [1], Corollary 1. 
Lemma 2.1. Let $w_{1}$ and $w_{2}$ be mesh function on a spatial mesh $\Omega_{h}$, vanishing at extreme points of $\widehat{\Omega_{h}}$ (the convex hull of $\Omega_{h}$ ) and satisfying $w_{1} \geq w_{2}$ on $\Omega_{h}$. Then we have the inequalities,

$$
\begin{aligned}
0 & \leq \sum_{x \in \Omega_{h}}\left(w_{1}\left|\chi_{w_{1}}(x)\right|-w_{2}(x)\left|\chi_{w_{2}}(x)\right|\right) \\
& \leq(n+1) \sum_{x \in \Omega_{h}}\left(w_{1}-w_{2}\right)(x)\left|\chi_{w_{1}}(x)\right| .
\end{aligned}
$$

In fact there is no loss of generality in replacing the functions $w_{1}$ and $w_{2}$ in Lemma 2.1 by their concave envelopes which then vanish on $\partial \widehat{\Omega_{h}}$. In this form, Lemma 2.1 is directly covered by Krylov [1].

Lemma 2.2. Let $w$ be a spatial mesh function on a mesh $\Omega \subset \mathbb{R}^{n}$, vanishing at extreme points of $\widehat{\Omega}$ (the convex hull of $\Omega$ ). Then for any $y \in \Omega$, we have the estimate,

$$
w(y) \leq\left\{\frac{d_{z}^{n}}{\omega_{n}} \sum_{x \in \Omega} w(x)\left|\chi_{w}(x)\right|\right\}^{\frac{1}{n+1}}
$$

where $d_{y}=\max _{x \in \Omega}|y-x|$.

Our purpose in this article is to deduce the discrete maximum principle in a form corresponding to (1.2), where the dependence on the coefficients of $L$ in (1.11) is determined by $\operatorname{det} A$ for $A$ given by (1.19). First we mention a lemma of [6]. Let

$$
V\left(y^{1}, \cdots, y^{n}\right)=\operatorname{det}\left[y_{j}^{i}\right]
$$

denote the volume of the parallelpiped spanned by $y^{1}, \cdots, y^{n}$.

Lemma 2.3. For $y^{1}, \cdots, y^{N} \in \mathbb{R}^{n}, N \geq n$, we have

$$
\operatorname{det}\left(\sum_{i=1}^{N} y^{i} \otimes y^{i}\right)=\sum_{1 \leq i_{1}<i_{2} \cdots<i_{n} \leq N} V^{2}\left(y^{i_{1}}, \cdots, y^{i_{n}}\right) .
$$

Moreover, for the difference operator $L$, given by (1.11), and the mesh $E$, we introduce a volume element $V(x, t)$ at a point $(x, t) \in E$ by

$$
V(x, t)=\max _{y^{1}, \cdots, y^{n} \in Z(x, t)} V\left(y^{1}-x, \cdots, y^{n}-x\right) .
$$

The volume element $V(x, t)$ is used in Theorem 3.1, instead of $h^{n}$ when uniform grids are considered with mesh length $h$. 


\section{Maximum principles}

In this section we prove an extension to general space-time meshes of our discrete maximum principle for parabolic differential operators [2]. We shall assume that the operator L, given by (1.11) is evolving, monotone, non-degenerate, in accordance with $(1.20),(1.22)$ and weakly positive, as in (1.23). We then associate non-negative constants $b_{o}^{\prime}$ and $c_{o}^{\prime}$ to the operator $L$ by assuming

$$
\begin{aligned}
b^{*}(x, t) & :=\sum_{s \leq t}\left|b^{\prime}(x, t, s)\right| \leq \tau \mu b_{o}^{\prime} \\
c^{\prime}(x, t) & :=\sum_{\substack{(y, s) \in E \\
s<t}} a(x, t ; y, s) \\
& \leq\left(1+c_{o}^{\prime} \tau\right) \mu .
\end{aligned}
$$

When $L$ is separately spatially balanced we clearly have $b_{o}^{\prime}=0$ and if $L$ is of positive type, (1.23) is satisfied for $\mu=c^{\prime}$ whence we can take $c_{o}^{\prime}=0$ in (3.2). We then have the following maximum principle.

Theorem 3.1. Let $u$ be a space-time mesh function satisfying the difference inequality,

$$
L u \geq f \quad \text { in } \mathfrak{D}^{o},
$$

together with the boundary condition

$$
u \leq 0 \quad \text { in } \mathfrak{D}^{b}
$$

Then we have the estimate

$$
\max _{\mathcal{D}} u \leq C d^{\frac{n}{n+1}}\left\{\sum_{(x, t) \in \mathcal{S}^{+}} \frac{|f(x, t)|^{n+1}}{\operatorname{det}[A(x, t)]} V(x, t) \tilde{\tau}(x, t)\right\}^{\frac{1}{n+1}},
$$

where $\tilde{\tau}(x, t)=\gamma^{-1}(x, t)$ and $C$ is a constant depending on $n, b_{o}^{\prime} T / d$ and $c_{o}^{\prime} T$.

Note that in $(3.4), V(x, t) \tilde{\tau}(x, t)$ interprets a volume element for the space-time mesh space $E$.

Proof. First we consider the case of spatially balanced operators of positive type, that is $b=0, c \leq 0$. We order the time values in $\mathfrak{D}$, by defining

$$
\begin{aligned}
& t_{o}=0, \\
& t_{i}=\min \left\{t \mid(x, t) \in \mathfrak{D}, \quad x \in \mathbb{R}^{n}, \quad t>t_{i-1}\right\}, \quad i=1,2, \ldots,
\end{aligned}
$$

so that

$$
0=t_{o}<t_{1} \ldots<t_{N}=T
$$


for some natural number $N$. We then define a finite sequence of spatial mesh functions $u_{o}, \ldots, u_{N}$ on corresponding discrete sets $\mathfrak{D}_{m} \subset \mathbb{R}^{n}$ by setting

$$
\begin{aligned}
u_{m}(x) & =u^{t_{m}}(x)=u\left(x, t_{m}\right), \\
\mathfrak{D}_{m} & =\mathfrak{D}_{t_{m}} .
\end{aligned}
$$

Writing

$$
\mathfrak{D}^{\prime}=\bigcup_{m=0}^{N} \mathfrak{D}_{m}
$$

we replace $\left\{u_{m}\right\}$ by a non-decreasing sequence $\left\{v_{m}\right\}$ of spatial mesh functions on $\mathfrak{D}^{\prime}$, by defining

$$
v_{m}(x)=\max _{\substack{x \in \mathcal{D}_{j} \\ j \leq m}}\left\{u_{j}(x), 0\right\} .
$$

At any point $x \in \mathfrak{D}^{\prime}$, where $v_{m}(x)>v_{m-1}(x)$, we have $v_{m}(x)=u_{m}(x)$. Consequently, setting

$$
\begin{aligned}
a_{m}^{\prime}(x, y) & =a^{\prime}\left(x, t_{m}, y\right), \\
c_{m}(x) & =c\left(x, t_{m}\right), \\
\gamma_{m}(x) & =\gamma\left(x, t_{m}\right), \\
f_{m}(x) & =f\left(x, t_{m}\right),
\end{aligned}
$$

we obtain from (3.3) and (1.12), the difference inequality,

$$
\begin{aligned}
L_{m}^{\prime} v_{m}(x) & \equiv \sum_{y \in \mathfrak{D}^{\prime}} a_{m}^{\prime}(x, y)\left(v_{m}(y)-v_{m}(x)\right) \\
& \quad+c_{m}(x) v_{m}(x)+\gamma_{m}(x)\left(v_{m-1}(x)-v_{m}(x)\right) \\
& \geq f_{m}(x) .
\end{aligned}
$$

Letting $\chi_{m}$ denote the normal mapping of the mesh function $v_{m}$ on $\mathfrak{D}^{\prime}$, that is

$$
\chi_{m}(x)=\left\{p \in \mathbb{R}^{n} \mid v_{m}(y) \leq v_{m}(x)+p \cdot(y-x) \text { for all } y \in \mathfrak{D}^{\prime}\right\},
$$

and

$$
\Gamma_{m}^{+}=\left\{x \in \mathfrak{D}^{\prime} \mid \chi_{m}(x) \neq \phi\right\},
$$

the upper contact set of $v_{m}$ on $\mathfrak{D}^{\prime}$, we suppose now that also $x \in \Gamma_{m}^{+}$and, following [3], [4], for some fixed $p \in \chi_{m}(x)$, set

$$
w_{m}(z)=v_{m}(z)-p \cdot(z-x),
$$


for $z \in \mathfrak{D}^{\prime}$. From (3.7), we then have

$$
\begin{aligned}
& \sum_{y \in \mathfrak{D}^{\prime}} a_{m}^{\prime}(x, y)\left(w_{m}(x)-w_{m}(y)\right)+\gamma_{m}(x)\left(v_{m}(x)-v_{m-1}(x)\right) \\
& \quad \leq \sum_{y \in \mathfrak{D}^{\prime}} a_{m}^{\prime}(x, y) p \cdot(x-y)+c_{m}(x) v_{m}(x)-f_{m}(x) \\
& \quad \leq-f_{m}(x)
\end{aligned}
$$

under our initial hypothesis that $L$ is balanced and positive. Following our treatment of the spatial case in [4], we define a function $\tilde{w}$ on the finite discrete set $Z_{m}=Z_{m}(x)=$ $Z\left(x, t_{m}\right)$, given by $(1.20)$, by

$$
\tilde{w}_{m}(z)=w_{m}(x)+a_{m}^{\prime}(x, y)\left(w_{m}(y)-w_{m}(x)\right)
$$

whenever

$$
z=z_{y}=x+a_{m}^{\prime}(x, y)(y-x)
$$

It follows that

$$
\chi_{m}(x)-p=\chi_{w_{m}}(x) \subset \chi_{\tilde{w}_{m}}(x)
$$

and moreover, from (3.9),

$$
\sum_{y \in \mathfrak{D}^{\prime}}\left(\tilde{w}_{m}(x)-\tilde{w}_{m}\left(z_{y}\right)\right) \leq-f_{m}(x)
$$

Let

$$
\left\{\begin{array}{l}
k(x)=1 \\
k(y)=0
\end{array}\right.
$$

We then have

$$
\begin{aligned}
\left|\chi_{\tilde{w}_{m}}\right| & \leq\left|f_{m}(x)\right|^{n}\left|\chi_{k}(x)\right| \\
& =\left|f_{m}(x)\right|^{n}\left|Z_{m}^{*}(x)\right|
\end{aligned}
$$

where $Z_{m}(x)=\left\{x+a^{\prime}\left(x, t_{m} ; y\right)(y-x) \mid y \in E^{\prime}(x, t)\right\}$ and

$$
\begin{aligned}
Z_{m}^{*}(x) & =\chi_{k}\left(x, t_{m}\right), \\
& \left.=\left\{p \in \mathbb{R}^{n} \mid p \cdot(y-x) \leq 1, \quad \text { for all } y \in Z_{m}(x)\right)\right\} .
\end{aligned}
$$

Moreover, we need a geometric inequality proved in Lemma 3 of [6],

$$
\left|Z_{m}^{*}(x)\right| \leq \frac{C}{\left|\widehat{Z_{m}}(x)\right|}
$$

where $C=n^{\frac{3 n}{2}} \omega_{n}^{2}$ and $\widehat{Z_{m}(x)}$ is the convex hull of $Z_{m}(x)$. From (3.14) and (3.11) we have

$$
\left|\chi_{m}(x)\right| \leq C(N) \frac{V\left(x, t_{m}\right)}{\operatorname{det}\left[A\left(x, t_{m}\right)\right]}\left|f_{m}(x)\right|^{n}
$$


where

$$
V\left(x, t_{m}\right)=\max _{y^{1}, \cdots, y^{n} \in Z_{m}(x)} V\left(y^{1}-x, \cdots, y^{n}-x\right) .
$$

From (3.9), we also have

$$
\left(v_{m}-v_{m-1}\right)(x) \leq \frac{f_{m}(x)}{\gamma_{m}(x)} .
$$

Therefore, we obtain

$$
\begin{aligned}
\sum_{x \in \mathfrak{D}} v_{m}\left|\chi_{m}(x)\right|-v_{m-1}\left|\chi_{m-1}(x)\right| & \leq(n+1) \sum_{x \in \mathfrak{D}}\left(v_{m}-v_{m-1}\right)(x)\left|\chi_{m}(x)\right| \\
& \leq C(n, N) \sum_{x \in \mathcal{S}_{m}^{+}} \frac{\left|f_{m}(x)\right|^{n+1} V\left(x, t_{m}\right)}{\gamma_{m}(x) \operatorname{det}\left[A\left(x, t_{m}\right)\right]}
\end{aligned}
$$

where

$$
\mathcal{S}_{m}^{+}=\left\{x \in \Gamma_{m}^{+} \mid v_{m}(x)>v_{m-1}(x)\right\} .
$$

Since $v_{0} \equiv 0$, we thus have

$$
\begin{aligned}
\sum_{x \in \mathfrak{D}} v_{m}\left|\chi_{m}(x)\right| & \leq C(n, N) \sum_{j=0}^{m} \sum_{x \in \mathcal{S}_{j}^{+}} \frac{\left|f_{j}(x)\right|^{n+1} V\left(x, t_{j}\right)}{\gamma_{j}(x) \operatorname{det}\left[A\left(x, t_{j}\right)\right]} \\
& \leq C(n, N) \sum_{(x, t) \in \mathcal{S}^{+}} \frac{|f(x, t)|^{n+1} V(x, t)}{\gamma(x, t) \operatorname{det}[A(x, t)]}
\end{aligned}
$$

Hence we conclude, from [2], Lemma 3.2,

$$
\begin{aligned}
\max _{\mathcal{D}} u & \leq \max _{\mathcal{P}^{\prime}} v_{N} \\
& \leq C(n, N) d^{\frac{n}{n+1}}\left\{\sum_{(x, t) \in \mathcal{S}^{+}} \frac{|f(x, t)|^{n+1}}{\operatorname{det}[A(x, t)]} V(x, t) \gamma^{-1}(x, t)\right\}^{\frac{1}{n+1}}
\end{aligned}
$$

and the estimate (3.4) in the case $b=0, c \leq 0$. by

To treat the general case, we introduce, as in [2], a modified mesh function $\bar{u}$ given

$$
\bar{u}=e^{-\kappa t} u(x, t),
$$

where $\kappa$ is a non-negative constant to be chosen later. For fixed $t \in\left\{t_{1}, \ldots, t_{N}\right\}$, we set

$$
\bar{t}=\min \left\{s \mid a(x, t ; y, s)>0, \quad \text { for some } x \in \mathfrak{D}_{t} \text { and }(y, s) \in \mathfrak{D}\right\}
$$

and define new coefficients $\bar{a}$ by

$$
\bar{a}(x, t ; y, s)=e^{\kappa(s-\bar{t})} a(x, t ; y, s) .
$$


Then we have the modified difference inequality,

$$
\begin{aligned}
\bar{L} \bar{u} & \equiv \sum \bar{a}(x, t ; y, s) \bar{u}(y, s) \\
& \geq e^{-\kappa \bar{t}} f(x, t) \\
& \geq-|f(x, t)|
\end{aligned}
$$

in place of (3.3). Furthermore, by (1.23)

$$
\begin{aligned}
\bar{c}(x, t) & =\sum_{(y, s) \in \mathfrak{D}} \bar{a}(x, t ; y, s) \\
& =\sum_{(y, t) \in \mathfrak{D}} e^{\kappa(t-\bar{t})} a(x, t ; y, t)+\sum_{\substack{(y, s) \in \mathfrak{D} \\
s<t}} e^{\kappa(s-\bar{t})} a(x, t ; y, s) \\
& \leq e^{\kappa(t-\tau-\bar{t})}\left\{-e^{\kappa \tau} \mu(x, t)+c^{\prime}(x, t)\right\} \\
& \leq 0
\end{aligned}
$$

provided

$$
\kappa \geq \frac{1}{\tau} \log \frac{c^{\prime}}{\mu} .
$$

Clearly, (1.23) is both necessary and sufficient for the operator $\bar{L}$ to be of positive type. Returning to the proof of Theorem 3.1, we replace $u$ by $\bar{u}$, with $v$ and $w$ defined accordingly, to obtain from (3.25), in place of (3.9),

$$
\begin{aligned}
& \sum_{y \in \mathfrak{D}^{\prime}} a_{m}^{\prime}(x, y)\left(w_{m}(x)-w_{m}(y)\right)+\gamma_{m}(x)\left(v_{m}(x)-v_{m-1}(x)\right) \\
& \quad \leq \sum_{y \in \mathfrak{D}^{\prime}} \bar{a}_{m}^{\prime}(x, y)\left(w_{m}(x)-w_{m}(y)\right)+\bar{\gamma}_{m}(x)\left(v_{m}(x)-v_{m-1}(x)\right) \\
& \quad \leq \bar{b}_{m}(x) \cdot p+\bar{c}_{m}(x) v_{m}(x)-e^{-\kappa \bar{t}} f_{m}(x)
\end{aligned}
$$

where

$$
\begin{aligned}
\bar{a}^{\prime}(x, y, t) & =\sum_{s \leq t} \bar{a}(x, t ; y, s), \\
\bar{\gamma}(x, t) & =\sum_{s<t} \bar{a}(x, t ; x, s), \\
\bar{b}(x, t) & =\sum \bar{a}(x, t ; y, s)(y-x),
\end{aligned}
$$

and

$$
\begin{aligned}
\bar{a}_{m}^{\prime}(x, y) & =\bar{a}^{\prime}\left(x, y, t_{m}\right), & \bar{\gamma}_{m}(x) & =\bar{\gamma}\left(x, t_{m}\right), \\
\bar{b}_{m}(x) & =\bar{b}\left(x, t_{m}\right), & \bar{c}_{m}(x) & =\bar{c}\left(x, t_{m}\right) .
\end{aligned}
$$


To handle the term involving $\bar{b}$ in (3.28), we proceed as in [5] and restrict the upper contact set and normal mapping of $v_{m}$ by extending $v_{m}$ to the ball $B=B_{R+\frac{1}{2} d}$ for some $R \geq 0$, so that $v_{m}$ vanishes in $B-\mathfrak{D}^{\prime}$. The normal mapping $\chi_{m}$ and upper contact set $\Gamma_{m}^{+}$are then defined with $B$ replacing $\mathfrak{D}^{\prime}$. We then have for any $p \in \chi_{m}(x)$,

$$
|p| \leq \frac{v_{m}(x)}{R},
$$

and hence we estimate, from (3.28)

$$
\begin{aligned}
& \sum_{y \in \mathfrak{D}^{\prime}} a_{m}^{\prime}(x, y)\left(w_{m}(x)-w_{m}(y)\right)+\gamma_{m}(x)\left(v_{m}(x)-v_{m-1}(x)\right) \\
& \quad \leq\left(\frac{\left|\bar{b}_{m}\right|}{R}+\bar{c}_{m}\right) v_{m}(x)+\left|f_{m}(x)\right| .
\end{aligned}
$$

Now, by (3.1) (3.2), we have

$$
\begin{aligned}
\frac{|\bar{b}(x, t)|}{R}+\bar{c}(x, t) & \leq \mu e^{\kappa(t-\tau-\bar{t})}\left\{\left(\frac{b_{o}^{\prime} \tau}{R}-1\right) e^{\kappa \tau}+1+c_{o}^{\prime} \tau\right\} \\
& \leq 0
\end{aligned}
$$

provided $\kappa$ and $R$ are chosen so that

$$
\left(1-\frac{b_{o}^{\prime} \tau}{R}\right) e^{\kappa \tau} \geq 1+c_{o}^{\prime} \tau
$$

Consequently we obtain, from (3.29)

$$
\sum_{y \in \mathfrak{D}^{\prime}} a_{m}^{\prime}(x, y)\left(w_{m}(x)-w_{m}(y)\right)+\gamma_{m}(x)\left(v_{m}(x)-v_{m-1}(x)\right) \leq\left|f_{m}(x)\right|
$$

and, as before, we conclude the estimate (3.22) with $u$ replaced by $\bar{u}$ and $d$ replaced by $d+R$. Accordingly, we have the estimate

$$
\max _{\mathcal{D}} u \leq C(n, N)(d+R)^{\frac{n}{n+1}} e^{\kappa T}\left\{\sum_{(x, t) \in \mathcal{S}^{+}} \frac{|f(x, t)|^{n+1}}{\operatorname{det}[A(x, t)]} \gamma^{-1} V(x, t)\right\}^{\frac{1}{n+1}}
$$

for any constants $\kappa$ and $R$ satisfying (3.30) in $\mathfrak{D}$. Choosing, say

$$
R=2 b_{o}^{\prime} T, \quad \kappa=1+2 c_{o}^{\prime},
$$

yields the estimate (3.4) as asserted. 


\section{References}

[1] N. V. Krylov, Sequence of convex functions and estimates of maximum of the solution of a parabolic equation, Sibirsk. Mat.Z. 17(1976), 290-303; Siberian Math. J. 17(1976), 226-237. [English translation]

[2] H. J. Kuo and N. S. Trudinger, On the discrete maximum principle for parabolic difference operators, RAIRO Modél. Math. Anal. Numér. 27(1993), 719-737.

[3] _ Positive difference operators on general meshes, Duke Math. J. 83(1996), 415-433.

[4] — Maximum principles for difference operators, Topics in Partial Differential Equations \& Applications: Collected papers in Honor of Carlo Pucci, Lecture Notes in Pure and Applied Mathematics Series/177, 209-219, Marcel Dekker, Inc. 1996.

[5] — Evolving monotone difference operators on general space-time meshes, Duke Math. J. 91(1998), 587-607.

[6] _ـ, A note on the discrete Aleksandrov-Bakelman maximum principle, Taiwanese J. of Math. 4(2000), 55-67.

Department of Applied Mathematics, National Chung-Hsing University, Taichung 402, Taiwan.

E-mail: kuohj@dragon.nchu.edu.tw

Centre for Mathematics and Its Applications, Australian National University, Canberra, ACT 0200, Australia.

E-mail: Neil.Trudinger@anu.edu.au 